\title{
Mitochondrial DNA Haplogroup JT is Related to Impaired Glycaemic Control and Renal Function in Type 2 Diabetic Patients
}

\author{
Noelia Diaz-Morales ${ }^{1}$ (1), Sandra Lopez-Domenech ${ }^{1}$, Francesca Iannantuoni ${ }^{1}$ (1), \\ Ester Lopez-Gallardo ${ }^{2,3,4}$, Eva Sola ${ }^{1}$, Carlos Morillas ${ }^{1}$, Milagros Rocha ${ }^{1,5, *}$, \\ Eduardo Ruiz-Pesini ${ }^{2,3,4,6}$ and Victor M. Victor ${ }^{1,5, *(D)}$ \\ 1 Service of Endocrinology, University Hospital Doctor Peset, Foundation for the Promotion of Health and \\ Biomedical Research in the Valencian Region (FISABIO), 46017 Valencia, Spain; \\ nodiazmo@alumni.uv.es (N.D.-M.); Sandra.lopez@uv.es (S.L.-D.); franian@alumni.uv.es (F.I.); \\ eva.sola@uv.es (E.S.); carlos.morillas@uv.es (C.M.) \\ 2 Department of Biochemistry and Molecular and Cell Biology, University of Zaragoza, 50013 Zaragoza, Spain; \\ esterlop@unizar.es (E.L.-G.); eduruiz@unizar.es (E.R.-P.) \\ 3 Instituto de Investigación Sanitaria de Aragón (IIS Aragón), 50013 Zaragoza, Spain \\ 4 Centro de Investigaciones Biomédicas En Red de Enfermedades Raras (CIBERER), 50013 Zaragoza, Spain \\ 5 CIBERehd-Department of Pharmacology and Physiology, University of Valencia, 46010 Valencia, Spain \\ 6 Fundación ARAID, 50018 Zaragoza, Spain \\ * Correspondence: victor.victor@uv.es (V.M.V.); milagros.rocha@uv.es (M.R.); Tel.: +34-961-622-757
}

Received: 17 July 2018; Accepted: 14 August 2018; Published: 16 August 2018

\begin{abstract}
The association between mitochondrial DNA (mtDNA) haplogroup and risk of type 2 diabetes (T2D) is undetermined and controversial. This study aims to evaluate the impact of the main mtDNA haplogroups on glycaemic control and renal function in a Spanish population of 303 T2D patients and 153 healthy controls. Anthropometrical and metabolic parameters were assessed and mtDNA haplogroup was determined in each individual. Distribution of the different haplogroups was similar in diabetic and healthy populations and, as expected, T2D patients showed poorer glycaemic control and renal function than controls. T2D patients belonging to the JT haplogroup (polymorphism m.4216T $>$ C) displayed statistically significant higher levels of fasting glucose and $\mathrm{HbA}_{1 \mathrm{c}}$ than those of the other haplogroups, suggesting a poorer glycaemic control. Furthermore, diabetic patients with the JT haplogroup showed a worse kidney function than those with other haplogroups, evident by higher levels of serum creatinine, lower estimated glomerular filtration rate (eGFR), and slightly higher (although not statistically significant) urinary albumin-to-creatinine ratio. Our results suggest that JT haplogroup (in particular, change at position 4216 of the mtDNA) is associated with poorer glycaemic control in T2D, which can trigger the development of diabetic nephropathy.
\end{abstract}

Keywords: type 2 diabetes mellitus; mitochondrial haplogroup; mtDNA; nephropathy; glycemic control

\section{Introduction}

Type 2 diabetes (T2D) has become one of the most common metabolic diseases, with a rapid increase in its prevalence over recent decades, representing an enormous cost to public health organisms. It is obvious that environmental factors—-such as diet and physical activity—play a key role in the pathogenesis of T2D, but an emerging body of evidence suggests that genetic factors can play an important role in the development and severity of T2D [1,2]. Therefore, characterization of new parameters that allow us 
to identify individuals at a high risk of developing T2D or to predict a poor prognostic of the disease are likely to be of great use in clinical practice, for designing strategies for primary prevention and for personalising treatments according to each specific condition.

Mitochondrial dysfunction is well known to be involved in the pathophysiology of T2D, as it affects not only insulin secretion but also insulin resistance [3,4]. In this sense, genetic factors such as mitochondrial DNA (mtDNA) variations may affect mitochondrial function and lead to the development of diabetes [5]. For example, an mtDNA mutation at nucleotide m.3243A $>\mathrm{G}$ has been described to cause maternally inherited diabetes and deafness [6,7]. Another relatively common variant, mtDNA m.16189T>C, has been associated with an enhanced risk of type 2 diabetes in Asian [8] and European [9] populations. Mitochondrial DNA haplogroups are defined by common variants of single nucleotide point (SNP) mutations of the mtDNA that result in a division of the population into discrete groups, each of which shares a common maternal ancestor. Although some studies have suggested mitochondrial haplogroups are involved in the genetic susceptibility of T2D [10-12], this connection is not altogether clear, as other authors have reported that haplogroups are unlikely to play a role in the risk of developing this disorder $[13,14]$.

Type 2 diabetes is characterised by inadequate metabolic control associated with subsequent microand macro-vascular complications. Fasting plasma glucose levels indicate how efficiently glucose levels are managed in the absence of dietary glucose, while glycated haemoglobin $\left(\mathrm{HbA}_{1 \mathrm{c}}\right)$ provides information regarding average blood glucose levels over the previous 8-12 weeks, thus representing an objective measurement of glycaemic control [15]. An association between poor glycaemic control and enhanced risk of microvascular complications, such as nephropathy, has been widely reported in diabetic patients [16,17]. Furthermore, some authors have suggested an effect of mtDNA haplogroups on the risk of developing diabetic complications in T2D [11,18,19]. However, whether or not mitochondrial haplogroups are involved in the glycaemic control of type 2 diabetic patients and the subsequent development of microvascular complications, such as nephropathy, has not yet been studied.

In the present study, we assessed a Spanish population of 303 T2D patients and 153 healthy controls with the aim of investigating differences in metabolic parameters and renal dysfunction markers according to the main mitochondrial macro-haplogroups.

\section{Experimental Section}

\subsection{Subjects}

Our study population was composed of 303 T2D patients and 153 healthy volunteers recruited at the Endocrinology and Nutrition Service of the University Hospital Dr. Peset (Valencia, Spain). T2D was diagnosed according to the criteria of the American Diabetes Association 2017 [20] (fasting plasma glucose $\geq 126 \mathrm{mg} / \mathrm{dL}$, or 2-h plasma glucose $\geq 200 \mathrm{mg} / \mathrm{dL}$ after a $75 \mathrm{~g}$ oral glucose tolerance test, or $\mathrm{HbA}_{1 \mathrm{C}} \geq 6.5 \%$, or random plasma glucose $\geq 200 \mathrm{mg} / \mathrm{dL}$ ). Subjects who met any of the following criterion were excluded from the study: history of cardiovascular disease (stroke, ischemic heart disease, peripheral vascular disease, and chronic disease related to cardiovascular risk); severe disease including malignances, autoimmune, inflammatory or infectious diseases; and abnormal haematological profile.

Written informed consent was obtained from all the participants before they participated in the study. The study was conducted in accordance with the Helsinki Declaration, and approved by the Ethics Committee of the University Hospital Dr. Peset (Project identification code: 97/16).

\subsection{Anthropometric and Biochemical Parameters}

During the medical appointment, weight $(\mathrm{kg})$, height $(\mathrm{m})$, systolic and diastolic blood pressure (SBP, DBP; $\mathrm{mm} \mathrm{Hg}$ ), and waist and hip circumference $(\mathrm{cm})$ were measured in all the participants. Body mass index (BMI; $\mathrm{kg} / \mathrm{m}^{2}$ ) and waist-to-hip ratio (WHR) were then calculated.

Venous blood samples were collected in fasting conditions from both control and type 2 diabetic subjects and centrifuged at $1500 \times \mathrm{g}$ for $10 \mathrm{~min}$ at $4{ }^{\circ} \mathrm{C}$ to obtain serum, in which levels of glucose, 
total cholesterol and triglycerides were determined by means of an automated enzymatic method using a Beckman Synchron LX20 Pro analyzer (Beckman Coulter, Brea, CA, USA). High-density lipoprotein cholesterol (HDL-c) levels were measured using a direct method with a Beckman Synchron LX20 Pro analyzer (Beckman Coulter, Brea, CA, USA), and low-density lipoprotein cholesterol (LDL-c) was calculated with Friedewald's formula. Insulin was measured with an Immulite 1000 automated immunoassay system (Siemens Healthcare SL, Madrid, Spain) and the homeostasis model assessment index of insulin resistance (HOMA-IR) was calculated to estimate insulin resistance using fasting insulin and glucose levels: HOMA $=$ [fasting insulin $(\mu \mathrm{U} / \mathrm{mL}) \times$ fasting glucose $(\mathrm{mg} / \mathrm{dL})] / 405$. HOMA index was only calculated for patients not undergoing insulin therapy. Percentage of $\mathrm{HbA}_{1 \mathrm{c}}$ was measured by means of an automatic glycohemoglobin analyzer (Arkray Inc., Kyoto, Japan) and high-sensitive C-reactive protein (hs-CRP) levels were assessed with the Dade Behring Nephelometer II Analyzer System using an immunonephelometric assay (Dade Behring, Deerfield, IL, USA).

Creatinine in serum and urine was determined by Jaffe's reaction. Measurements of urinary albumin concentrations were performed by turbidimetry with an Architect c-16000 autoanalyzer (Abbott, Lake Bluff, IL, USA). Estimated glomerular filtration rate (eGFR) was calculated by the CKD-EPI equation from serum creatinine [21].

\subsection{Haplotyping}

Total DNA was extracted from whole blood with the REALPURE "SSS" Kit (Durviz SL, Valencia, Spain) and stored at $-20^{\circ} \mathrm{C}$ until analysis.

Mitochondrial haplogroups HV, JT and U were defined by the mtDNA polymorphisms m.7028C $>\mathrm{T}$, m.12308A $>$ G, m.4216T $>C$ and m.14766T $>C$ [22]. These haplogroups encompass around $90 \%$ of the Spanish population [23]. Samples revealing other haplogroups with low frequencies among the population (those not classified as HV, JT or U) were grouped altogether and referred to as "Others". Custom designed Taqman ${ } S N P$ genotyping assays (Applied Biosystems, Foster City, CA, USA) were used to analyse mtDNA genetic variants and samples were run in a Step One Plus Real Time PCR System (Applied Biosystems, Foster City, CA, USA). The analysis consisted of a pre-read and post-read step of the plate of $30 \mathrm{~s}$ at $60^{\circ} \mathrm{C}$, before and after the PCR cycle. The cycle conditions were $10 \mathrm{~min}$ at $95^{\circ} \mathrm{C}$, followed by 40 cycles of $15 \mathrm{~s}$ at $95^{\circ} \mathrm{C}$ and $1 \mathrm{~min}$ at $60^{\circ} \mathrm{C}$. Information on the haplogroups, dyes, probes and primers in each assay is widely explained in Nogales-Gadea et al. [24]. For each genotype analysis, positive and negative controls from different previously characterised mtDNA aliquots were used to ensure an adequate internal control.

\subsection{Statistical Analysis}

Results were processed using SPSS Software version 17.0 (SPSS Statistics Inc., Chicago, IL, USA) for statistical analysis. Data in tables are presented as means \pm standard deviation for normally distributed data, medians (25th and 75th quartile) for non-normally distributed data, or percentage for qualitative variables. Figures show mean and standard error of the mean. Potential differences between haplogroups were analysed by ANOVA for normally distributed variables and the Kruskal-Wallis test for non-normally distributed variables. When differences among groups were detected, Student-Newman-Keuls or Dunn's multiple-comparison post hoc test were applied, as appropriate. Frequencies in T2D patients and control subjects were compared using the chi-square test. A Student's $t$-test was employed to evaluate differences between controls and type 2 diabetic patients. The effect of possible covariates (such as age, sex, BMI or duration of diabetes) was analyzed with a univariate general linear model. For all the tests, a two-tailed $p<0.05$ was considered significant.

\section{Results}

\subsection{Clinical Characteristics of the Study Population}

Our observational study included 303 type 2 diabetic patients and 153 healthy controls. Haplogroup distribution, and anthropometrical and inflammatory characteristics, as well as lipid profile of the studied participants are shown in Table 1. 
Table 1. Haplogroup distribution and characteristics of the study population. Anthropometrical, inflammatory and lipid profile.

\begin{tabular}{|c|c|c|c|c|c|c|c|c|c|c|c|c|c|}
\hline \multirow[b]{2}{*}{ Haplogroup } & \multicolumn{6}{|c|}{ Healthy Controls } & \multicolumn{6}{|c|}{ Type 2 Diabetic Patients } & \multirow{2}{*}{$p$-value } \\
\hline & HV & JT & $\mathrm{U}$ & Others & Total & $p$-value & HV & JT & $\mathrm{U}$ & Others & Total & $p$-value & \\
\hline $\mathrm{N}(\%)$ & $81(52.9)$ & $26(17.0)$ & $35(22.9)$ & $11(7.2)$ & $153(100)$ & & $165(54.5)$ & $43(14.2)$ & $65(21.4)$ & $30(9.9)$ & $303(100)$ & - & 0.68 \\
\hline Male \% & 37.0 & 41.9 & 23.7 & 27.3 & 34.2 & 0.36 & 68.5 & 81.8 & 77.3 & 66.7 & 72.1 & 0.22 & $<0.001$ \\
\hline Age (years) & $41.3 \pm 16.4$ & $45.3 \pm 16.0$ & $41.5 \pm 15.7$ & $40.0 \pm 14.0$ & $41.9 \pm 16.0$ & 0.68 & $58.6 \pm 9.8$ & $59.5 \pm 9.3$ & $58.4 \pm 9.9$ & $59.3 \pm 7.8$ & $58.8 \pm 9.5$ & 0.92 & $<0.001$ \\
\hline $\operatorname{BMI}\left(\mathrm{kg} / \mathrm{m}^{2}\right)$ & $24.4 \pm 3.7$ & $25.6 \pm 4.5$ & $25.2 \pm 4.2$ & $23.7 \pm 2.3$ & $24.8 \pm 24.8$ & 0.39 & $30.8 \pm 4.8$ & $30.2 \pm 3.8$ & $30.1 \pm 4.4$ & $31.2 \pm 4.2$ & $30.6 \pm 4.5$ & 0.68 & $<0.001$ \\
\hline WHR & $0.83 \pm 0.08$ & $0.84 \pm 0.13$ & $0.85 \pm 0.09$ & $0.79 \pm 0.08$ & $0.83 \pm 0.09$ & 0.41 & $0.98 \pm 0.08$ & $0.97 \pm 0.06$ & $1.00 \pm 0.08$ & $0.98 \pm 0.07$ & $0.98 \pm 0.08$ & 0.58 & $<0.001$ \\
\hline $\mathrm{SBP}(\mathrm{mmHg})$ & $122 \pm 18$ & $123 \pm 21$ & $122 \pm 18$ & $116 \pm 16$ & $122 \pm 18$ & 0.80 & $133 \pm 18$ & $132 \pm 15$ & $135 \pm 18$ & $137 \pm 18$ & $134 \pm 17$ & 0.79 & $<0.001$ \\
\hline $\mathrm{DBP}(\mathrm{mmHg})$ & $73 \pm 10$ & $73 \pm 10$ & $77 \pm 14$ & $72 \pm 11$ & $74 \pm 11$ & 0.45 & $78 \pm 10$ & $79 \pm 11$ & $79 \pm 9$ & $77 \pm 11$ & $78 \pm 10$ & 0.88 & $<0.001$ \\
\hline Duration of diabetes & & & & & - & - & $10.1 \pm 8.4$ & $9.7 \pm 6.7$ & $11.0 \pm 8.5$ & $10.6 \pm 6.7$ & $10.2 \pm 8.0$ & 0.87 & - \\
\hline Hs-CRP (mg/L) & $\begin{array}{c}0.82 \\
(0.45-2.23)\end{array}$ & $\begin{array}{c}1.07 \\
(0.40-3.16)\end{array}$ & $\begin{array}{c}1.04 \\
(0.45-2.86)\end{array}$ & $\begin{array}{c}1.24 \\
(0.31-2.60)\end{array}$ & $\begin{array}{c}1.01 \\
(0.45-2.60)\end{array}$ & 0.76 & $\begin{array}{c}2.57 \\
(1.19-5.76)\end{array}$ & $\begin{array}{c}2.34 \\
(1.09-5.24)\end{array}$ & $\begin{array}{c}2.56 \\
(0.95-5.61)\end{array}$ & $\begin{array}{c}2.53 \\
(1.04-5.20)\end{array}$ & $\begin{array}{c}2.53 \\
(1.08-5.50)\end{array}$ & 0.86 & $<0.001$ \\
\hline TC (mg/dL) & $189 \pm 35$ & $194 \pm 29$ & $190 \pm 42$ & $203 \pm 32$ & $191 \pm 36$ & 0.62 & $180 \pm 41$ & $181 \pm 45$ & $170 \pm 36$ & $182 \pm 33$ & $178 \pm 40$ & 0.35 & 0.001 \\
\hline HDL-c (mg/dL) & $55.9 \pm 16.5$ & $54.5 \pm 16.0$ & $54.7 \pm 11.6$ & $59.9 \pm 13.1$ & $55.7 \pm 15.1$ & 0.76 & $42.8 \pm 11.0$ & $41.9 \pm 11.4$ & $41.4 \pm 9.5$ & $44.3 \pm 9.5$ & $42.5 \pm 10.6$ & 0.62 & $<0.001$ \\
\hline LDL-c (mg/dL) & $115 \pm 31$ & $122 \pm 28$ & $118 \pm 35$ & $125 \pm 24.9$ & $118 \pm 30.9$ & 0.67 & $109 \pm 36.0$ & $108 \pm 37.3$ & $100 \pm 33.1$ & $109 \pm 32.4$ & $107 \pm 35.3$ & 0.34 & 0.002 \\
\hline Triglycerides (mg/dL) & $69(51-114)$ & $70(47-104)$ & $75(58-94)$ & $86(60-117)$ & $73(54-104)$ & 0.63 & $122(88-176)$ & $\begin{array}{c}123 \\
(101-156)\end{array}$ & $129(94-200)$ & $\begin{array}{c}134 \\
(101-172)\end{array}$ & $125(93-174)$ & 0.94 & $<0.001$ \\
\hline
\end{tabular}

Normally distributed data are shown as mean $\pm \mathrm{SD}$ and non-normally distributed data as median (25th-75th quartiles). $p$-value * when comparing type 2 diabetic patients (Total) vs. healthy controls (Total). Abbreviations: BMI, body-mass index; DBP, diastolic blood pressure; HDL-c, high-density lipoprotein cholesterol; hs-CRP, high-sensitive C-reactive protein; LDL-c, low-density lipoprotein cholesterol; SBP, systolic blood pressure; SD, standard deviation; TC, total cholesterol; WHR, waist-to-hip ratio. 
Our cohort of healthy controls showed a haplogroup distribution similar to that reported in a larger Spanish population by Dahmany et al. [23]. No differences were found in the distribution of the different macro-haplogroups between control subjects and diabetic patients $(p=0.68)$. Although our cohort of T2D patients was characterised by higher age and percentage of men with respect to the control population $(p<0.001)$, when sub-classified by haplogroup, no differences were found in these parameters among haplogroups in the diabetic population ( $p=0.92$ for age and $p=0.22$ for male percentage) and control subjects ( $p=0.68$ for age and $p=0.36$ for male percentage). As expected, T2D patients in total had higher body mass index (BMI), waist-to-hip ratio (WHR), systolic blood pressure (SBP), diastolic blood pressure (DBP), high-sensitive C-reactive protein (hs-CRP) $(p<0.001)$ than control subjects. Lipid profile in the diabetic patients showed typical characteristics of atherogenic dyslipidemia, with elevated levels of triglycerides ( $p<0.001$ vs. control) and low levels of HDL-c $(p<0.001$ when compared to control subjects). The lower levels of total cholesterol $(p=0.001)$ and LDL-c $(p=0.002)$ found in diabetic patients vs. controls were probably due to the fact that most of the patients were being treated with antihyperlipidemic agents (Table 2). No statistically significant differences in the studied parameters were detected according to haplogroup in the type 2 diabetic population or the control group (see Table 1 for $p$-values).

Pharmacologic treatment of the type 2 diabetic patients included in this study is shown in Table 2. No significant differences were observed between the percentages of patients treated with hypolipidemic, antidiabetic, and antihypertensive agents (see Table 2 for $p$-values).

Table 2. Pharmacological treatment of the study's cohort of type 2 diabetic patients.

\begin{tabular}{ccccccc}
\hline & HV & JT & U & Others & Total & $p$-value \\
\hline Statins (\%) & 56.5 & 50.0 & 53.2 & 56.0 & 54.7 & 0.91 \\
Fibrate (\%) & 13.0 & 19.4 & 8.5 & 12.0 & 13.0 & 0.54 \\
Ezetimibe (\%) & 3.2 & 5.0 & 6.7 & 0.0 & 4.0 & 0.73 \\
Metformin (\%) & 63.8 & 83.3 & 77.6 & 60.0 & 69.5 & 0.06 \\
DPP-4 inhibitors (\%) & 33.9 & 45.0 & 41.9 & 42.9 & 38.6 & 0.76 \\
Insulin (\%) & 29.3 & 19.4 & 30.6 & 20.0 & 27.0 & 0.51 \\
Sulfonilureas (\%) & 18.8 & 14.3 & 10.4 & 33.3 & 17.8 & 0.11 \\
Glitazones (\%) & 7.8 & 19.4 & 4.1 & 12.0 & 9.3 & 0.09 \\
Glinides (\%) & 21.6 & 16.7 & 8.2 & 32.0 & 19.0 & 0.07 \\
GLP-1 agonists (\%) & 19.4 & 15.0 & 6.5 & 21.4 & 15.7 & 0.40 \\
Antihypertensive (\%) & 45.2 & 43.2 & 49.0 & 48.0 & 46.0 & 0.95 \\
\hline
\end{tabular}

Abbreviations: DPP-4, dipeptidil peptidasa-4; GLP-1, glucagon-like peptide-1.

\subsection{Glucose Metabolism}

First analysis performed was a comparison between T2D patients and controls as a whole, without subdividing by haplogroup (Table A1). As expected, type 2 diabetic patients showed higher levels of fasting glucose, $\mathrm{HbA}_{1 \mathrm{c}}$, fasting insulin and HOMA-IR index than control subjects $(p<0.001)$. Differences between controls and subjects with T2D remained statistically significant after adjustment for age, sex, and BMI ( $p<0.001$ for glucose, $\mathrm{HbA}_{1 \mathrm{c}}$ and HOMA; $p=0.01$ for insulin). Graphs showing parameters related with glycaemic control and insulin resistance are plotted in Figure 1. Differences in glucose, $\mathrm{HbA1c}$, insulin, and HOMA between control subjects and T2D patients remained significant after subdivided by haplogroup ( $p<0.001$ for glucose, HbA1c, and HOMA when comparing control vs T2D belonging to haplogroups HV, JT, $\mathrm{U}$, and Others. For insulin levels: $p<0.001$ when comparing control vs T2D in haplogroup HV; $p<0.01$ in haplogroup JT; and $p<0.05$ in haplogroups U and Others. Differences in $p$-values found in the levels of insulin are attributable to differences in the sample size between haplogroups).

Interestingly, diabetic patients with the JT haplogroup showed significantly higher levels of fasting glucose $(p=0.001)$ and $\mathrm{HbA}_{1 \mathrm{c}}(p=0.007)$ compared to patients belonging to the other haplogroups analysed (grey bars in Figure 1A,B). These differences remained statistically significant 
despite adjustments for duration of diabetes ( $p=0.006$ for fasting glucose and $p=0.002$ for $\mathrm{HbA}_{1 \mathrm{c}}$ ). Nevertheless, no differences were found in the levels of fasting insulin $(p=0.50)$ and HOMA-IR index $(p=0.38)$ when T2D patients with different haplogroups were compared (grey bars in Figure 1C,D). Control subjects did not reveal differences depending on haplogroup for any of the parameters related with glycaemic control and insulin resistance (white bars in Figure 1; glucose: $p=0.70 ; \mathrm{HbA}_{1 \mathrm{c}}: p=0.81$; insulin: $p=0.60$; HOMA-IR: $p=0.83$ ).
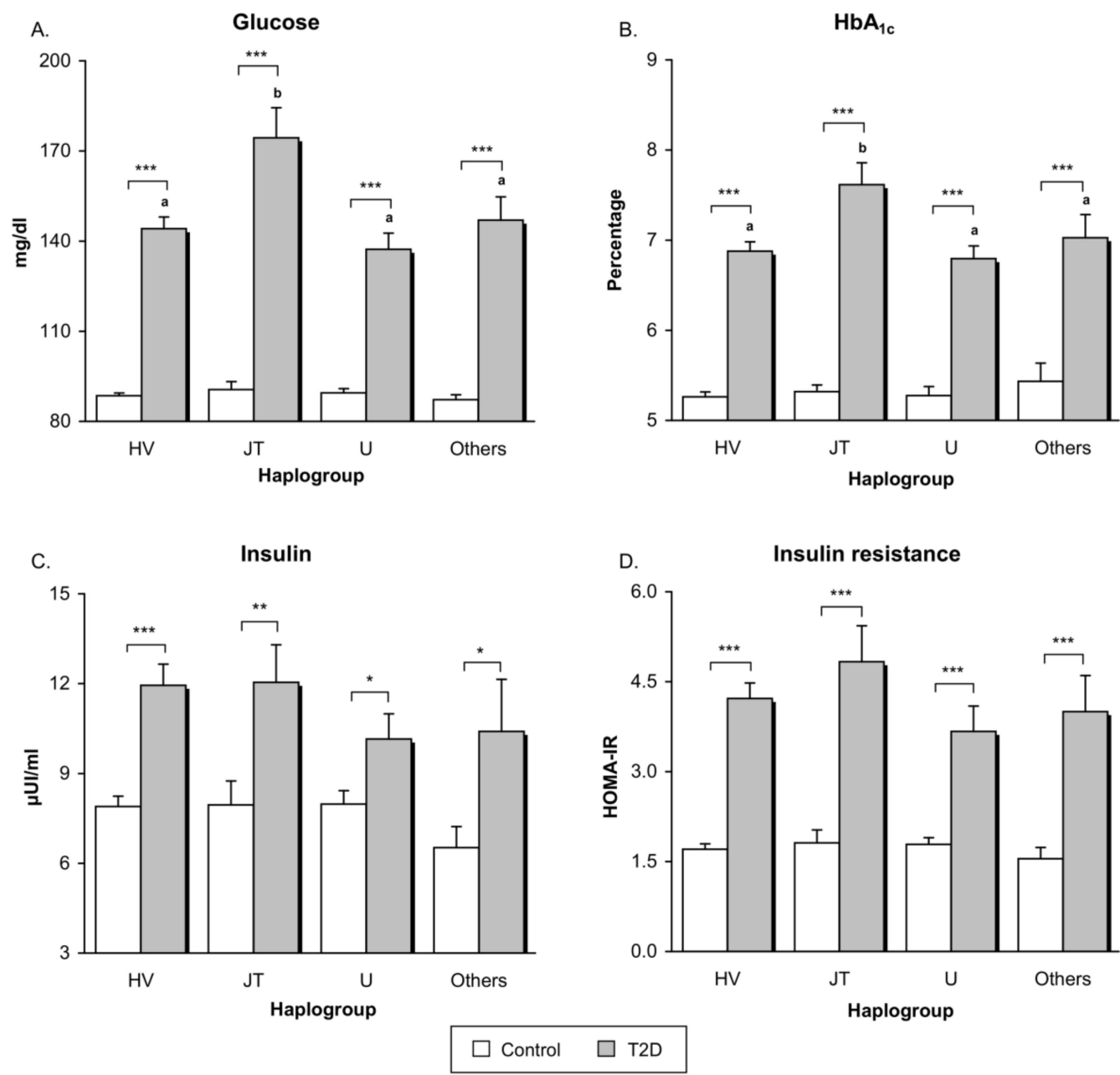

Figure 1. Glycaemic control and insulin resistance-related parameters in healthy controls and type 2 diabetic patients belonging to the main mitochondrial macro-haplogroups. (A) Serum levels of fasting glucose (expressed as mg/dL); (B) Percentage of glycated haemoglobin (HbA1c) levels; (C) Serum levels of fasting insulin $(\mu \mathrm{UI} / \mathrm{mL})$; (D) HOMA-IR index calculated as [fasting insulin $(\mu \mathrm{U} / \mathrm{mL}) \times$ fasting glucose $(\mathrm{mg} / \mathrm{dL})] / 405$. Only patients not treated with insulin therapy are included in the graphs of fasting insulin and HOMA-IR. Grey bars show type 2 diabetic patients and white bars represent controls. ${ }^{*} p<0.05 ;{ }^{* *} p<0.01 ;{ }^{* *} p<0.001$ when compared controls vs. type 2 diabetic subjects. Letters indicate significant differences among type 2 diabetic patients with different haplogroups $(p<0.05)$ when compared by means of one-way ANOVA followed by Student-Newman-Keuls post-hoc test (i.e., bars tagged with the same letter do not differ significantly from each other, while bars with no letter in common are significantly different from each other $(p<0.05))$. Abbreviations: HbA1c, glycated haemoglobin; HOMA-IR, Homeostasis model assessment index of insulin resistance; T2D, Type 2 diabetes. 


\subsection{Renal Function}

Type 2 diabetic patients, when analyzed as a whole, exhibited lower kidney function than control subjects, expressed by higher serum creatinine concentrations $(p<0.001)$ and lower eGFR $(p<0.001)$ (Appendix Table A1). Differences in eGFR between controls and patients remained when adjusted by age, sex, and BMI $(p=0.04)$, whereas differences in creatinine levels were not longer statistically significant after adjusting for age, sex, and BMI $(p=0.12)$. After subdivided by haplogroup, differences between T2D patients and controls remained statistically significant only in haplogroups HV $(p<0.001)$ and JT $p<0.05$ for serum creatinine, and in haplogroups HV $(p<0.01), \operatorname{JT}(p<0.05)$, and Others $(p<0.05)$ for eGFR. Differences in $p$-values found between the different haplogroups are probably due to differences in the sample size.

In the case of T2D patients, those with the JT haplogroup showed a worse renal function than patients belonging to $\mathrm{HV}, \mathrm{U}$, and Others haplogroups, manifested as significantly higher levels of serum creatinine (grey bars in Figure 2A, $p<0.001$ ) and lower eGFR (grey bars in Figure 2B, $p=0.01$ ). Differences between the JT group and all the other macro-haplogroups in creatinine levels and eGFR did not change in the diabetic population after adjusting by duration of diabetes $(p<0.001$ for creatinine levels and $p=0.003$ for eGFR). Control subjects did not reveal statistically significant differences in kidney function according to haplogroups (white bars in Figure 2; $p=0.09$ for creatinine and $p=0.27$ for eGRF).
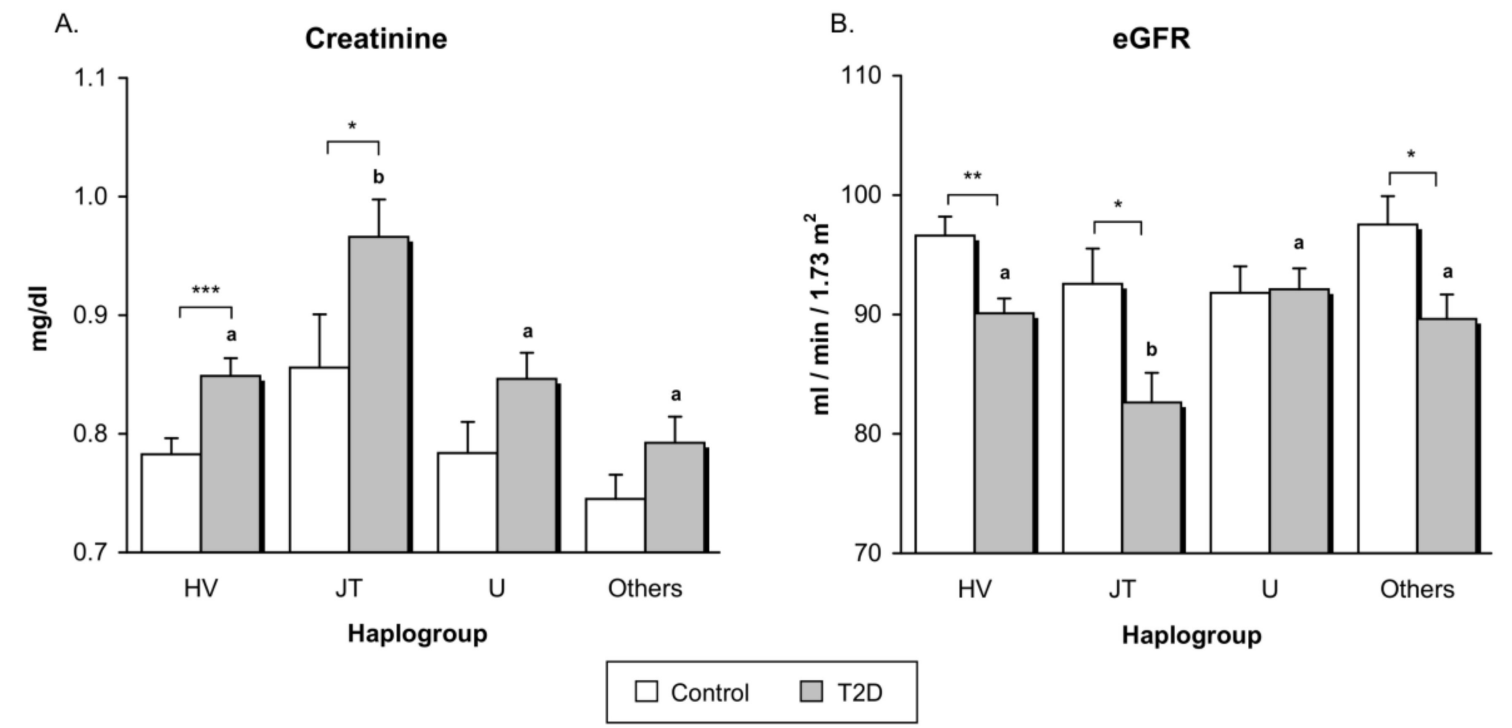

Figure 2. Assessment of renal function in healthy volunteers and type 2 diabetic patients subdivided by mitochondrial haplogroup. (A) Concentrations of serum creatinine; (B) Levels of estimated glomerular filtration rate (eGFR). White bars correspond to controls, while grey bars represent type 2 diabetic patients. ${ }^{*} p<0.05 ;{ }^{* *} p<0.01 ;{ }^{* * *} p<0.001$ in controls vs. type 2 diabetic subjects. Letters indicate significant differences among type 2 diabetic patients with different haplogroups $(p<0.05)$ when compared by means of one-way ANOVA followed by Student-Newman-Keuls post-hoc test (i.e., bars tagged with the same letter do not differ significantly from each other, while bars with no letter in common are significantly different from each other, $(p<0.05))$. Abbreviations: eGFR, estimated glomerular filtration rate; T2D, Type 2 diabetes.

In light of the above mentioned results, we also analyzed concentrations of urinary albumin and creatinine in patients in whom said parameters were measured on the same day as blood was extracted; namely, in a total of 106 type 2 diabetic patients (52 from the HV group, 16 from the JT group, 26 from the U group, and 12 from the "Others" group). Urinary albumin-to-creatinine ratio $(\mathrm{mg} / \mathrm{g})$ was slightly higher in the JT group $(19.46 \pm 17.54 \mathrm{mg} / \mathrm{g})$ than in those belonging to the other 
haplogroups (HV: $12.55 \pm 7.92 \mathrm{mg} / \mathrm{g}$; U: $12.54 \pm 8.73 \mathrm{mg} / \mathrm{g}$; others: $12.49 \pm 7.33 \mathrm{mg} / \mathrm{g}$ ), although it did not reach statistical significance in the one-way ANOVA test $(p=0.099)$.

\section{Discussion}

In the present study, we have performed a case-control study to explore the possible effects of the main mitochondrial haplogroups on metabolic control and renal function in a Spanish population of 303 type 2 diabetic patients and 153 healthy controls. We have observed that T2D patients belonging to the JT macrohaplogroup showed enhanced levels of fasting plasma glucose, $\mathrm{HbA1c}$, creatinine, and decreased eGFR when compared to patients from the other haplogroups ( $\mathrm{HV}, \mathrm{U}$, and Others), thus suggesting poorer metabolic control and renal function in T2D patients with the JT haplogroup.

Mitochondria are responsible for the cell's energy supply through oxidative phosphorylation (OXPHOS), and some of the proteins involved in this process are encoded in the mtDNA. Given the importance of OXPHOS in insulin secretion [25,26], different genetic variants are potential candidates for playing a role in the susceptibility to or protection against metabolic defects [13]. Mitochondrial haplogroups are clusters of phylogenetically related mtDNA haplotypes that might have been selected during evolution to permit humans to adapt to famine or cold climates [27]. It has been suggested that these mtDNA variants contribute to energy metabolism and, hence, may be associated with metabolic diseases [28]. Crispim et al. [10] reported that the European-specific JT mitochondrial haplogroup was associated with insulin resistance and type 2 diabetes in Caucasian-Brazilian patients, as patients belonging to the JT cluster exhibited higher levels of HOMA-IR. In addition, the J1 haplogroup is thought to be involved in susceptibility to type 2 diabetes among Caucasian (Jewish) patients depending on family health history [29]. According to several studies performed in Asian populations, individuals carrying haplogroup N9a are less susceptible to type 2 diabetes and metabolic syndrome [30,31]. However, in spite of this evidence, the association between mitochondrial haplogroups and type 2 diabetes is not clear, with many studies providing conflicting results or failing to find significant associations $[13,14,18,32]$. Our results do not show a direct association of the development of T2D with the main macro-haplogroups, as no differences were found in the frequencies of each haplogroup between our diabetic and control populations. Interestingly, we found that patients belonging to the JT cluster presented poorer glycaemic control and higher levels of fasting glucose and $\mathrm{HbA1c}$, than other patients, thus suggesting that said haplogroup is involved in the metabolism of glucose in patients with T2D. Our findings are in agreement with those reported by Crispim et al. [10] which described higher levels of HOMA-IR in patients with JT haplogroup, although no statistically significant differences were found in our cohort of type 2 diabetic patients, probably because the size of our sample was smaller than the sample size in the cited work.

Type 2 diabetes and inadequate glycaemic control are frequently associated with macro- and micro-vascular complications. Whether or not mitochondrial haplogroups play a role in modulating the development of T2D-related complications is a question that has been widely studied. Achilli et al. [18] found an association of various mitochondrial haplogroups and increased risk of diabetic complications in an Italian population: haplogroup $\mathrm{H} 3$ increased the probability of developing neuropathy; haplogroup $\mathrm{H}$ was linked to retinopathy; and subjects harbouring $\mathrm{V}$ and $\mathrm{U} 3 \mathrm{mtDNA}$ showed enhanced incidence of renal failure and nephropathy. In this context, it is worth pointing out that diabetic nephropathy has been associated with specific mitochondrial haplogroups in several studies; for instance, Feder et al. [33] reported a link with the J1 haplogroup in an Ashkenazi Jewish population, while Niu et al. [11] reported a link with the N9a haplogroup in a Chinese population. Our data are in accordance with an involvement of mitochondrial haplogroup in the development of nephropathy, as our type 2 diabetic patients belonging to the JT haplogroup showed higher levels of serum creatinine and lower levels of eGFR compared to patients belonging to the other haplogroups analyzed. Interestingly, though not statistically significant, T2D patients harbouring the JT haplogroup also presented a higher urinary albumin-to-creatinine ratio. Taken together, these results suggest T2D patients with the JT haplogroup are likely to have impaired kidney function. 
The variant $\mathrm{m} .4216 \mathrm{~T}>\mathrm{C}$, a key SNP for defining the JT macro-haplogroup [34], leads to a non-synonymous amino acid change in the mtDNA MT-ND1 gene encoding NADH:Ubiquinone oxidoreductase core subunit 1 (p.MT-ND1), one of the components of the mitochondrial respiratory complex I. Electrons coming from glucose metabolism through glycolysis and the Krebs cycle are principally stored in NADH for ATP production and oxygen reduction. It has been proposed that hyperglycaemia can increase the production of the complex I substrate NADH [35]. Overproduction of NADH leads to an electron pressure on the mitochondrial electron transport chain that drives to an increase in electron leakage and the subsequent high production of reactive oxygen species (ROS) $[36,37]$. As the major enzyme implicated in NADH recycling, mitochondrial complex I impairment can lead to further increased levels of NADH [38], with the following enhancement of ROS. Altogether these mechanisms will induce oxidative stress, which has been widely reported as a central player in the development of insulin resistance, pancreatic $\beta$-cells dysfunction, and finally, type 2 diabetes [39-41]. A recent study has demonstrated that pharmacological inhibition of complex I of mitochondrial electron transport chain improves glucose homeostasis and ameliorates hyperglycaemia [42]. Our findings bring us to hypothesize that variant $\mathrm{m} .4216 \mathrm{~T}>\mathrm{C}$ results in aberrant activity of complex I that, under diabetic conditions, leads to poorer glycaemic control. For this reason, we suggest that inhibitors of complex I—such as metformin and thiazolidinediones [43]—may be adequate drugs for the treatment of T2D patients belonging to the JT haplogroup.

Both hyperglycaemia and excessive oxidative stress are well known to be involved in the development of diabetic vascular complications [44,45], including microvascular complications such as nephropathy [46]. The kidney is especially vulnerable to the damage produced by hyperglycaemia-induced oxidative stress; in fact said damage has been suggested as an important mechanism involved in the pathogenesis of tubular and glomerular abnormalities [46].

The present study has some limitations in terms of statistical power. We did not perform a previous sample size estimation due to the lack of studies addressing the role of the haplogroups here studied (HV, JT, U, and others) on metabolic control and renal function. However, we consider that our study has enough power for reaching statistically significant differences among different haplogroups in the diabetic population. Nevertheless, researches in larger populations will serve to confirm these results.

Together, this evidence leads us to hypothesize that the JT haplogroup (in particular, change at position 4216 of the mtDNA) might result in a poorer glycaemic control in type 2 diabetic patients, thus contributing to the development of diabetic nephropathy. Future studies with a larger sample size would help to confirm our results and, if corroborated, haplogroup screening in recently diagnosed T2D patients might be suggested as a way of predicting disease progression and choosing the most adequate clinical treatment for avoiding macro and microvascular-associated complications.

Author Contributions: Conceptualization, V.M.V., M.R., and E.R.-P; Methodology, N.D.-M., F.I., S.L.-D. and E.L.-G.; Validation, V.M.V., E.R.-P., M.R., and N.D.-M.; Formal Analysis, N.D.-M. and M.R.; Investigation, N.D.-M., F.I., S.L.-D., and E.L.-G.; Resources, V.M.V., E.R.-P., E.S., and C.M.; Data Curation, N.D.-M. and F.I.; Writing-Original Draft Preparation, N.D.-M. and V.M.V.; Writing-Review and Editing, V.M.V., M.R., E.R.-P., N.D.-M., F.I., S.L.-D., E.L.-G., E.S., and C.M.; Visualization, N.D.-M. and M.R.; Supervision, V.M.V., M.R., and E.R.-P.; Project Administration, V.M.V., M.R., and E.R.-P.; Funding Acquisition, V.M.V., M.R., and E.R.-P.

Funding: This research was funded by grants from Carlos III Health Institute (ISCIII) (PI16/1083, PI16/0301, PI14/00070, PI17/00166); CIBERehd (CB06/04/0071) and CIBERER-initiatives of the ISCIII; the European Regional Development Fund (ERDF "A way to build Europe"); and Departamento de Ciencia, Tecnología y Universidad del Gobierno de Aragón (Grupos de Referencia B33_17R); Unrestricted grant from Menarini S.A.; N.D.-M. and S.L.-D. are recipient of PFIS contracts from Carlos III Health Institute (FI14/00125 and FI14/00350, respectively). F.I. has a contract from Generalitat Valenciana (GRISOLIAP/2016/015). V.M.V. and M.R. are recipients of contracts from the Ministry of Health of the Valencian Regional Government and Carlos III Health Institute (CES10/030 and CPII16/00037, respectively).

Acknowledgments: The authors thank Brian Normanly (University of Valencia-CIBERehd) for his editorial assistance, and Rosa Falcon (FISABIO) for her technical assistance.

Conflicts of Interest: The authors declare no conflict of interest. The funders had no role in the design of the study; in the collection, analyses, or interpretation of data; in the writing of the manuscript, and in the decision to publish the results. 


\section{Appendix}

Table A1. Differences in glycaemic control and renal function between controls and type 2 diabetic patients.

\begin{tabular}{ccccc}
\hline & Healthy Controls & $\begin{array}{c}\text { Type 2 Diabetic } \\
\text { Patients }\end{array}$ & $p$-value & $\begin{array}{c}\text { Age, Sex, and BMI } \\
\text { Adjusted } \boldsymbol{p} \text {-value }\end{array}$ \\
\hline Glucose $(\mathrm{mg} / \mathrm{dL})$ & $89.04 \pm 8.93$ & $147.39 \pm 50.87$ & $<0.001$ & $<0.001$ \\
HbA $1 \mathrm{c}(\%)$ & $5.28 \pm 0.32$ & $6.99 \pm 1.32$ & $<0.001$ & $<0.001$ \\
Insulin $(\mu \mathrm{HI} / \mathrm{mL})$ & $7.84 \pm 2.98$ & $11.50 \pm 5.91$ & $<0.001$ & 0.013 \\
HOMA-IR & $1.73 \pm 0.76$ & $4.21 \pm 2.39$ & $<0.001$ & $<0.001$ \\
Creatinine $(\mathrm{mg} / \mathrm{dL})$ & $0.79 \pm 0.12$ & $0.86 \pm 0.17$ & $<0.001$ & 0.12 \\
eGFR $\left(\mathrm{mL} / \mathrm{min} / 1.73 \mathrm{~m}^{2}\right)$ & $94.92 \pm 9.93$ & $89.39 \pm 13.19$ & $<0.001$ & 0.04 \\
\hline
\end{tabular}

Data are shown as mean \pm SD. Abbreviations: BMI, body-mass index; eGFR, estimated glomerular filtration rate; HbA1c, glycated haemoglobin; HOMA-IR: Homeostasis model assessment index of insulin resistance; SD: standard deviation.

\section{References}

1. Ali, O. Genetics of type 2 diabetes. World J. Diabetes 2013, 4, 114-123. [CrossRef] [PubMed]

2. Hara, K.; Shojima, N.; Hosoe, J.; Kadowaki, T. Genetic architecture of type 2 diabetes. Biochem. Biophys. Res. Commun. 2014, 452, 213-220. [CrossRef] [PubMed]

3. Supale, S.; Li, N.; Brun, T.; Maechler, P. Mitochondrial dysfunction in pancreatic beta cells. Trends Endocrinol. Metab. 2012, 23, 477-487. [CrossRef] [PubMed]

4. Gonzalez-Franquesa, A.; Patti, M.E. Insulin Resistance and Mitochondrial Dysfunction. Adv. Exp. Med. Biol. 2017, 982, 465-520. [CrossRef] [PubMed]

5. Kwak, S.H.; Park, K.S. Role of mitochondrial DNA variation in the pathogenesis of diabetes mellitus. Front. Biosci. (Landmark Ed.) 2016, 21, 1151-1167. [PubMed]

6. Van den Ouweland, J.M.; Lemkes, H.H.; Ruitenbeek, W.; Sandkuijl, L.A.; de Vijlder, M.F.; Struyvenberg, P.A.; van de Kamp, J.J.; Maassen, J.A. Mutation in mitochondrial tRNA(Leu)(UUR) gene in a large pedigree with maternally transmitted type II diabetes mellitus and deafness. Nat. Genet. 1992, 1, 368-371. [CrossRef] [PubMed]

7. Reardon, W.; Ross, R.J.; Sweeney, M.G.; Luxon, L.M.; Pembrey, M.E.; Harding, A.E.; Trembath, R.C. Diabetes mellitus associated with a pathogenic point mutation in mitochondrial DNA. Lancet 1992, 340, 1376-1379. [CrossRef]

8. Park, K.S.; Chan, J.C.; Chuang, L.M.; Suzuki, S.; Araki, E.; Nanjo, K.; Ji, L.; Ng, M.; Nishi, M.; Furuta, H.; et al. Study group of molecular diabetology in Asia A mitochondrial DNA variant at position 16189 is associated with type 2 diabetes mellitus in Asians. Diabetologia 2008, 51, 602-608. [CrossRef] [PubMed]

9. Ye, Z.; Gillson, C.; Sims, M.; Khaw, K.T.; Plotka, M.; Poulton, J.; Langenberg, C.; Wareham, N.J. The association of the mitochondrial DNA OriB variant (16184-16193 polycytosine tract) with type 2 diabetes in Europid populations. Diabetologia 2013, 56, 1907-1913. [CrossRef] [PubMed]

10. Crispim, D.; Canani, L.H.; Gross, J.L.; Tschiedel, B.; Souto, K.E.; Roisenberg, I. The European-specific mitochondrial cluster $\mathrm{J} / \mathrm{T}$ could confer an increased risk of insulin-resistance and type 2 diabetes: An analysis of the m.4216T >C and m.4917A >G variants. Ann. Hum. Genet. 2006, 70, 488-495. [CrossRef] [PubMed]

11. Niu, Q.; Zhang, W.; Wang, H.; Guan, X.; Lu, J.; Li, W. Effects of mitochondrial haplogroup N9a on type 2 diabetes mellitus and its associated complications. Exp. Ther. Med. 2015, 10, 1918-1924. [CrossRef] [PubMed]

12. Jiang, W.; Li, R.; Zhang, Y.; Wang, P.; Wu, T.; Lin, J.; Yu, J.; Gu, M. Mitochondrial DNA Mutations Associated with Type 2 Diabetes Mellitus in Chinese Uyghur Population. Sci. Rep. 2017, 7, 16989. [CrossRef] [PubMed]

13. Saxena, R.; de Bakker, P.I.; Singer, K.; Mootha, V.; Burtt, N.; Hirschhorn, J.N.; Gaudet, D.; Isomaa, B.; Daly, M.J.; Groop, L.; et al. Comprehensive association testing of common mitochondrial DNA variation in metabolic disease. Am. J. Hum. Genet. 2006, 79, 54-61. [CrossRef] [PubMed]

14. Chinnery, P.F.; Mowbray, C.; Patel, S.K.; Elson, J.L.; Sampson, M.; Hitman, G.A.; McCarthy, M.I.; Hattersley, A.T.; Walker, M. Mitochondrial DNA haplogroups and type 2 diabetes: A study of 897 cases and 1010 controls. J. Med. Genet. 2007, 44, e80. [CrossRef] [PubMed]

15. Nathan, D.M.; Turgeon, H.; Regan, S. Relationship between glycated haemoglobin levels and mean glucose levels over time. Diabetologia 2007, 50, 2239-2244. [CrossRef] [PubMed] 
16. Klein, R. Hyperglycemia and microvascular and macrovascular disease in diabetes. Diabetes Care 1995, 18, $258-268$. [CrossRef] [PubMed]

17. Stratton, I.M.; Adler, A.I.; Neil, H.A.; Matthews, D.R.; Manley, S.E.; Cull, C.A.; Hadden, D.; Turner, R.C.; Holman, R.R. Association of glycaemia with macrovascular and microvascular complications of type 2 diabetes (UKPDS 35): Prospective observational study. BMJ 2000, 321, 405-412. [CrossRef] [PubMed]

18. Achilli, A.; Olivieri, A.; Pala, M.; Hooshiar Kashani, B.; Carossa, V.; Perego, U.A.; Gandini, F.; Santoro, A.; Battaglia, V.; Grugni, V.; et al. Mitochondrial DNA backgrounds might modulate diabetes complications rather than T2DM as a whole. PLoS ONE 2011, 6, e21029. [CrossRef] [PubMed]

19. Estopinal, C.B.; Chocron, I.M.; Parks, M.B.; Wade, E.A.; Roberson, R.M.; Burgess, L.G.; Brantley, M.A., Jr.; Samuels, D.C. Mitochondrial haplogroups are associated with severity of diabetic retinopathy. Invest. Ophthalmol. Vis. Sci. 2014, 55, 5589-5595. [CrossRef] [PubMed]

20. American Diabetes Association 2. Classification and Diagnosis of Diabetes. Diabetes Care 2017, 40, S11-S24. [CrossRef] [PubMed]

21. Levey, A.S.; Stevens, L.A.; Schmid, C.H.; Zhang, Y.L.; Castro, A.F., 3rd; Feldman, H.I.; Kusek, J.W.; Eggers, P.; Van Lente, F.; Greene, T.; et al. CKD-EPI (Chronic Kidney Disease Epidemiology Collaboration) A new equation to estimate glomerular filtration rate. Ann. Intern. Med. 2009, 150, 604-612. [CrossRef] [PubMed]

22. Van Oven, M.; Kayser, M. Updated comprehensive phylogenetic tree of global human mitochondrial DNA variation. Hum. Mutat. 2009, 30, E386-E394. [CrossRef] [PubMed]

23. Dahmany, Y.; Marcuello, A.; Montiel-Sosa, F.; Martínez-Redondo, D.; Montoya, J.; Díez-Sánchez, C.; López-Pérez, M.; Ruiz-Pesini, E. Mitochondrial lineages distribution in the Spanish population: Anticipating association studies. An. R. Acad. Nac. Farm. 2006, 72, 37-47.

24. Nogales-Gadea, G.; Pinos, T.; Ruiz, J.R.; Marzo, P.F.; Fiuza-Luces, C.; Lopez-Gallardo, E.; Ruiz-Pesini, E.; Martin, M.A.; Arenas, J.; Moran, M.; et al. Are mitochondrial haplogroups associated with elite athletic status? A study on a Spanish cohort. Mitochondrion 2011, 11, 905-908. [CrossRef] [PubMed]

25. Olsson, A.H.; Yang, B.T.; Hall, E.; Taneera, J.; Salehi, A.; Nitert, M.D.; Ling, C. Decreased expression of genes involved in oxidative phosphorylation in human pancreatic islets from patients with type 2 diabetes. Eur. J. Endocrinol. 2011, 165, 589-595. [CrossRef] [PubMed]

26. Sharoyko, V.V.; Abels, M.; Sun, J.; Nicholas, L.M.; Mollet, I.G.; Stamenkovic, J.A.; Gohring, I.; Malmgren, S.; Storm, P.; Fadista, J.; et al. Loss of TFB1M results in mitochondrial dysfunction that leads to impaired insulin secretion and diabetes. Hum. Mol. Genet. 2014, 23, 5733-5749. [CrossRef] [PubMed]

27. Mishmar, D.; Ruiz-Pesini, E.; Golik, P.; Macaulay, V.; Clark, A.G.; Hosseini, S.; Brandon, M.; Easley, K.; Chen, E.; Brown, M.D.; et al. Natural selection shaped regional mtDNA variation in humans. Proc. Natl. Acad. Sci. USA 2003, 100, 171-176. [CrossRef] [PubMed]

28. Ruiz-Pesini, E.; Mishmar, D.; Brandon, M.; Procaccio, V.; Wallace, D.C. Effects of purifying and adaptive selection on regional variation in human mtDNA. Science 2004, 303, 223-226. [CrossRef] [PubMed]

29. Feder, J.; Ovadia, O.; Blech, I.; Cohen, J.; Wainstein, J.; Harman-Boehm, I.; Glaser, B.; Mishmar, D. Parental diabetes status reveals association of mitochondrial DNA haplogroup J1 with type 2 diabetes. BMC Med. Genet. 2009, 10, 60. [CrossRef] [PubMed]

30. Fuku, N.; Park, K.S.; Yamada, Y.; Nishigaki, Y.; Cho, Y.M.; Matsuo, H.; Segawa, T.; Watanabe, S.; Kato, K.; Yokoi, K.; et al. Mitochondrial haplogroup N9a confers resistance against type 2 diabetes in Asians. Am. J. Hum. Genet. 2007, 80, 407-415. [CrossRef] [PubMed]

31. Tanaka, M.; Fuku, N.; Nishigaki, Y.; Matsuo, H.; Segawa, T.; Watanabe, S.; Kato, K.; Yokoi, K.; Ito, M.; Nozawa, Y.; et al. Women with mitochondrial haplogroup N9a are protected against metabolic syndrome. Diabetes 2007, 56, 518-521. [CrossRef] [PubMed]

32. Mohlke, K.L.; Jackson, A.U.; Scott, L.J.; Peck, E.C.; Suh, Y.D.; Chines, P.S.; Watanabe, R.M.; Buchanan, T.A.; Conneely, K.N.; Erdos, M.R.; et al. Mitochondrial polymorphisms and susceptibility to type 2 diabetes-related traits in Finns. Hum. Genet. 2005, 118, 245-254. [CrossRef] [PubMed]

33. Feder, J.; Blech, I.; Ovadia, O.; Amar, S.; Wainstein, J.; Raz, I.; Dadon, S.; Arking, D.E.; Glaser, B.; Mishmar, D. Differences in mtDNA haplogroup distribution among 3 Jewish populations alter susceptibility to T2DM complications. BMC Genomics 2008, 9, 198. [CrossRef] [PubMed] 
34. Herrnstadt, C.; Elson, J.L.; Fahy, E.; Preston, G.; Turnbull, D.M.; Anderson, C.; Ghosh, S.S.; Olefsky, J.M.; Beal, M.F.; Davis, R.E.; et al. Reduced-median-network analysis of complete mitochondrial DNA coding-region sequences for the major African, Asian, and European haplogroups. Am. J. Hum. Genet. 2002, 70, 1152-1171. [CrossRef] [PubMed]

35. Kabat, A.; Ponicke, K.; Salameh, A.; Mohr, F.W.; Dhein, S. Effect of a beta 2-adrenoceptor stimulation on hyperglycemia-induced endothelial dysfunction. J. Pharmacol. Exp. Ther. 2004, 308, 564-573. [CrossRef] [PubMed]

36. Vinogradov, A.D.; Grivennikova, V.G. Oxidation of NADH and ROS production by respiratory complex I. Biochim. Biophys. Acta 2016, 1857, 863-871. [CrossRef] [PubMed]

37. Yan, L.J. Pathogenesis of chronic hyperglycemia: From reductive stress to oxidative stress. J. Diabetes Res. 2014, 2014, 137919. [CrossRef] [PubMed]

38. He, Q.; Wang, M.; Petucci, C.; Gardell, S.J.; Han, X. Rotenone induces reductive stress and triacylglycerol deposition in C2C12 cells. Int. J. Biochem. Cell Biol. 2013, 45, 2749-2755. [CrossRef] [PubMed]

39. Evans, J.L.; Goldfine, I.D.; Maddux, B.A.; Grodsky, G.M. Are oxidative stress-activated signaling pathways mediators of insulin resistance and beta-cell dysfunction? Diabetes 2003, 52, 1-8. [CrossRef] [PubMed]

40. Shah, S.; Iqbal, M.; Karam, J.; Salifu, M.; McFarlane, S.I. Oxidative stress, glucose metabolism, and the prevention of type 2 diabetes: Pathophysiological insights. Antioxid. Redox Signal. 2007, 9, 911-929. [CrossRef] [PubMed]

41. Rocha, M.; Rovira-Llopis, S.; Banuls, C.; Bellod, L.; Falcon, R.; Castello, R.; Morillas, C.; Herance, J.R.; Hernandez-Mijares, A.; Victor, V.M. Mitochondrial dysfunction and oxidative stress in insulin resistance. Curr. Pharm. Des. 2013, 19, 5730-5741. [CrossRef] [PubMed]

42. Hou, W.L.; Yin, J.; Alimujiang, M.; Yu, X.Y.; Ai, L.G.; Bao, Y.Q.; Liu, F.; Jia, W.P. Inhibition of mitochondrial complex I improves glucose metabolism independently of AMPK activation. J. Cell. Mol. Med. 2018, 22, 1316-1328. [CrossRef] [PubMed]

43. Brunmair, B.; Staniek, K.; Gras, F.; Scharf, N.; Althaym, A.; Clara, R.; Roden, M.; Gnaiger, E.; Nohl, H.; Waldhausl, W.; et al. Thiazolidinediones, like metformin, inhibit respiratory complex I: A common mechanism contributing to their antidiabetic actions? Diabetes 2004, 53, 1052-1059. [CrossRef] [PubMed]

44. Nishikawa, T.; Edelstein, D.; Du, X.L.; Yamagishi, S.; Matsumura, T.; Kaneda, Y.; Yorek, M.A.; Beebe, D.; Oates, P.J.; Hammes, H.P.; Giardino, I.; Brownlee, M. Normalizing mitochondrial superoxide production blocks three pathways of hyperglycaemic damage. Nature 2000, 404, 787-790. [CrossRef] [PubMed]

45. Giacco, F.; Brownlee, M. Oxidative stress and diabetic complications. Circ. Res. 2010, 107, 1058-1070. [CrossRef] [PubMed]

46. Singh, D.K.; Winocour, P.; Farrington, K. Oxidative stress in early diabetic nephropathy: Fueling the fire. Nat. Rev. Endocrinol. 2011, 7, 176-184. [CrossRef] [PubMed]

(C) 2018 by the authors. Licensee MDPI, Basel, Switzerland. This article is an open access article distributed under the terms and conditions of the Creative Commons Attribution (CC BY) license (http://creativecommons.org/licenses/by/4.0/). 